\title{
Talleres de reanimación neonatal en la Sociedad Mexicana de Pediatría durante la pandemia por SARS-CoV-2
}

\section{Neonatal resuscitation workshops at Sociedad Mexicana de Pediatría during the SARS-CoV-2 pandemic}

\author{
Claudia Montesinos-Ramírez,* José Luis Pinacho-Velázquez,* Silvia F Torres-Lira,* \\ Alicia Elizabeth Robledo-Galván,* Pedro Iván Martínez-Barrera,* Erika Karina Quiroz-Mendieta,* \\ Erika Corral-Kassian,* Dillan David Izaguirre-Alcántara,* Sergio Carrillo-Arteaga*
}

* Sociedad Mexicana de Pediatría. México.

A finales de la década de 1990, los miembros de la Organización de las Naciones Unidas y otras entidades internacionales acordaron los Objetivos de Desarrollo del Milenio. Uno de estos Objetivos fue la reducción de la mortalidad infantil, proponiendo disminuir en dos terceras partes la mortalidad en niños menores de cinco años. Para esos años, con respecto a la mortalidad neonatal, aproximadamente un tercio de las defunciones se presentaban en las primeras 24 horas de vida, y cerca de tres cuartas partes en la primera semana. Con las estrategias para cumplir los Objetivos del Milenio, el número de muertes de recién nacidos $(\mathrm{RN})$ en el ámbito mundial descendió de cinco millones en 1990 a 2.4 millones en 2019. Para este descenso, los programas de reanimación neonatal han sido ampliamente reconocidos como una de las acciones que influyeron de manera determinante.

La Sociedad Mexicana de Pediatría (SMP), desde su fundación, se ha interesado en los problemas de salud pública, por lo que ha realizado sus actividades para coadyuvar en beneficio de la salud infantil. En particular en el tema de RN, desde el año 2002 ha ofrecido talleres de Reanimación Neonatal (RENEO), tanto para proveedores como para la formación de instructores.
Estos talleres presenciales se realizan con apego a guías internacionales, por lo cual, los participantes que los acreditan tienen reconocimiento por la Secretaría de Salud de México durante dos a tres años; tras este periodo, se debe volver a cursar a fin de obtener la recertificación. Debido a los avances en la investigación, las guías se modifican, por lo que el programa de los talleres RENEO se actualiza en consecuencia, tomando en cuenta los lineamientos de la Academia Americana de Pediatría (AAP), la Asociación Americana del Corazón (AHA) y del Comité de Enlace Internacional sobre Reanimación (ILCOR). Además, estos talleres son ofrecidos por un equipo de instructores de alto nivel, bajo el liderazgo de médicos pediatras comprometidos con la educación médica continua. Todo lo anterior ha sido favorecedor para que, de manera progresiva, se incremente el número de profesionales de la salud (médicos, enfermeras, residentes y estudiantes) que se han capacitado en las instalaciones de la SMP.

Sin embargo, estos logros tuvieron una interrupción por la pandemia por COVID-19, suspendiéndose desde marzo del 2020 los talleres RENEO de manera presencial. Como todos hemos sido testigos, la pandemia por SARS-CoV-2 ha impactado seriamente tanto

Correspondencia: Claudia Montesinos-Ramírez, claumontesinos@hotmail.com

Citar como: Montesinos-Ramírez C, Pinacho-Velázquez JL, Torres-Lira SF, Robledo-Galván AE, Martínez-Barrera PI, Quiroz-Mendieta EK et al. Talleres de reanimación neonatal en la Sociedad Mexicana de Pediatría durante la pandemia por SARS-CoV-2. Rev Mex Pediatr. 2021; 88(2): 51-52. https://dx.doi.org/10.35366/101277 
a estos talleres como a otras actividades académicas tradicionales, por lo que para mantener la educación médica continua se ha optado por la educación médica a distancia. Si bien la SMP ha desarrollado e implementado cursos en línea para adaptarse a la nueva realidad, esta modalidad no parece ser suficiente 0 apropiada para actividades educativas que incluyen el desarrollo de habilidades y destrezas de los asistentes, como los talleres RENEO. Lo anterior debido a que parte del contenido de los talleres incluye la evaluación y retroalimentación a los participantes, tomando en cuenta las acciones realizadas.

Por esto, y en virtud de que la pandemia continúa, la SMP en el Centro de Adiestramiento en Reanimación Pediátrica (CARP) desde abril del 2021 reinició los talleres de RENEO de manera híbrida. El equipo de instructores y directivos del CARP diseñaron el programa que brinda un ambiente de confianza y seguridad entre alumnos e instructores. En esta modalidad híbrida, los participantes reciben vía electrónica los documentos para la capacitación, después existen sesiones virtuales interactivas con los instructores y, por último, se lleva a cabo la evaluación de conocimientos, también a distancia. Quienes acreditan la parte teórica, acuden al CARP para la sesión de evaluación de las habilidades de manera presencial. Pero antes de acudir, se debe comprobar que tanto instructores como alumnos estén libres de COVID-19, mediante un cuestionario sobre síntomas, antecedentes de vacunación y de contacto con pacientes/familiares con COVID-19.
El taller se lleva a cabo con un limitado número de asistentes, donde se prioriza el distanciamiento y el uso de equipo de protección personal. Por fortuna, hasta el momento la respuesta ha sido muy entusiasta por parte de instructores y de los interesados en la capacitación, quienes se sienten muy satisfechos, por lo cual seguiremos programando los talleres RENEO bajo esta modalidad.

$\mathrm{Al}$ adaptarnos a la nueva realidad, la SMP mantiene el espíritu y los objetivos planteados desde su fundación. Estamos orgullosos de los talleres RENEO, porque estamos convencidos de que contribuimos con el abatimiento de la mortalidad neonatal en México, así como de las secuelas por asfixia perinatal y sus complicaciones.

El equipo de instructores, directivos y administrativos del CARP y de la SMP reconocemos que aún resta camino por recorrer y que iremos haciendo modificaciones para mejorar la estrategia educativa, con la finalidad de ofrecer a los profesionales de la salud un modelo de capacitación con técnicas innovadoras, desarrolladas por expertos en materia de educación, salud y tecnología.

Amor y ciencia al servicio de la niñez.

Conflicto de intereses: Los autores declaran que no tienen. 